\title{
PRINCIPAL STRESS AND STRAIN TRAJECTORIES IN NONLINEAR ELASTOSTATICS*
}

BY

R. W. OGDEN

University of Glasgow

\begin{abstract}
The Maxwell-Lamé equations governing the principal components of Cauchy stress for plane deformations are well known in the context of photo-elasticity, and they form a pair of coupled first-order hyperbolic partial differential equations when the deformation geometry is known. In the present paper this theme is developed for nonlinear isotropic elastic materials by supplementing the (Lagrangean form of the) equilibrium equations by a pair of compatibility equations governing the deformation. The resulting equations form a system of four first-order partial differential equations governing the principal stretches of the plane deformation and the two angles which define the orientation of the Lagrangean and Eulerian principal axes of the deformation. Coordinate curves are chosen to coincide locally with the Lagrangean (Eulerian) principal strain trajectories in the undeformed (deformed) material.

Coupled with appropriate boundary conditions these equations can be used to calculate directly the principal stretches and stresses together with their trajectories. The theory is illustrated by means of a simple example.
\end{abstract}

1. Introduction. In plane linear elasticity the equilibrium equations in the absence of body forces may be written in the form

$$
\begin{aligned}
& \frac{\partial \sigma_{1}}{\partial s_{\xi}}+\frac{\left(\sigma_{1}-\sigma_{2}\right)}{\rho_{\eta}}=0, \\
& \frac{\partial \sigma_{2}}{\partial s_{\eta}}+\frac{\left(\sigma_{1}-\sigma_{2}\right)}{\rho_{\xi}}=0,
\end{aligned}
$$

where $\sigma_{1}, \sigma_{2}$ are the in-plane principal stresses, $(\xi, \eta)$ are (orthogonal) curvilinear coordinates corresponding to coordinate directions coinciding locally with the in-plane principal directions of stress, $\rho_{\xi}, \rho_{\eta}$ are the radii of curvature of the coordinate curves $\eta=$ constant and $\xi=$ constant respectively, and $s_{\xi}, s_{\eta}$ are associated arclength parameters.

${ }^{*}$ Received September 27, 1984. 
If $\theta$ denotes the direction of the tangent to the coordinate curves $\eta=$ constant relative to the $x_{1}$-axis of an in-plane rectangular Cartesian coordinate system $\left(x_{1}, x_{2}\right)$, then

$$
\tan 2 \theta=\frac{2 \sigma_{12}}{\sigma_{11}-\sigma_{22}}
$$

where $\sigma_{\alpha \beta}(\alpha, \beta=1,2)$ are the Cartesian components of the stress tensor. We also have

$$
\frac{1}{\rho_{\xi}}=\frac{\partial \theta}{\partial s_{\xi}}, \quad \frac{1}{\rho_{\eta}}=\frac{\partial \theta}{\partial s_{\eta}} .
$$

The (orthogonal) coordinate transformation between $\left(x_{1}, x_{2}\right)$ and $(\xi, \eta)$ satisfies

$$
\begin{array}{ll}
\frac{\partial x_{1}}{\partial s_{\xi}}=\cos \theta, & \frac{\partial x_{1}}{\partial s_{\eta}}=-\sin \theta, \\
\frac{\partial x_{2}}{\partial s_{\xi}}=\sin \theta, & \frac{\partial x_{2}}{\partial s_{\eta}}=\cos \theta,
\end{array}
$$

or, equivalently,

$$
\begin{array}{ll}
\frac{\partial s_{\xi}}{\partial x_{1}}=\cos \theta, & \frac{\partial s_{\xi}}{\partial x_{2}}=\sin \theta, \\
\frac{\partial s_{\eta}}{\partial x_{1}}=-\sin \theta, & \frac{\partial s_{\eta}}{\partial x_{2}}=\cos \theta .
\end{array}
$$

For an isotropic elastic material, equation (2) is coupled with

$$
\tan 2 \theta=\frac{2 e_{12}}{e_{11}-e_{22}}
$$

where $e_{\alpha \beta}(\alpha, \beta=1,2)$ are the Cartesian components of the infinitesimal strain tensor (whose principal directions then coincide with those of the stress tensor).

Equations (1) are known as the Maxwell-Lamé equations and they are used as a basis for comparing experimental results with theory in the context of photoelasticity; see, for example [1]. Assuming that $\theta, \rho_{\xi}, \rho_{\eta}$ and the principal strains are known from experimental measurements, equations (1) serve to determine the principal stresses $\sigma_{1}, \sigma_{2}$ and hence the stress trajectories. Thus the properties of an isotropic elastic material can be assessed in nonhomogeneous deformations. In this framework the hyperbolic character of equations (1) has been remarked upon in [2].

Clearly, equations (1) apply to any material in equilibrium in the absence of body forces, as also do Eqs. (2)-(5). In particular, they apply in nonlinear elasticity.

The objective of the present paper is first to provide a Lagrangean formulation of the equilibrium equations, analogous to (1), for nonlinear elastic materials and secondly to supplement these with appropriate compatibility equations. The resulting system of four equations with four dependent variables forms a first-order system (not, in general, hyperbolic). For any given nonlinear isotropic elastic constitutive law the equations may be solved for the deformation when suitable boundary conditions are prescribed. 
The specialization of the above-mentioned compatibility conditions to the case of linear isotropic elasticity yields a second-order equation coupling $\theta$ with the principal infinitesimal strains $e_{1}, e_{2}$. With equations (1) and Hooke's Law this forms a system of three equations for $e_{1}, e_{2}$, and $\theta$.

The formulation of the equations provided here is particularly suited to the calculation of stress and strain trajectories in a deformed elastic material. It has the advantage that it requires the constitutive law of an isotropic elastic material to be expressed in terms of the principal stretches of the deformation (which have immediate physical interpretations). Moreover, the equations are in a form which facilitates the numerical computation of solutions to boundary-value problems.

The use of the equations is illustrated by their application to a simple problem whose solution does not require a numerical treatment. From the computational viewpoint the equations and boundary conditions have some novel features, and it is appropriate to deal with these in a separate paper.

2. Deformation and stress. Let $\mathscr{B}_{0} \subset \mathscr{E}^{3}$, where $\mathscr{E}^{3}$ denotes a three-dimensional Euclidean space, be the region occupied by the considered material body in some reference configuration. Let $\chi: \mathscr{B}_{0} \rightarrow \mathscr{B} \subset \mathscr{E}^{3}$ denote the deformation of the body from $\mathscr{B}_{0}$ onto the region $\mathscr{B}$ in some current configuration. We label points in $\mathscr{B}_{0}$ and $\mathscr{B}$ by their position vectors $\mathbf{X}$ and $\mathbf{x}$ respectively relative to an appropriate choice of origin, so that

$$
\mathbf{x}=\mathbf{x}(\mathbf{X}), \quad \mathbf{X} \in \mathscr{B}_{0} .
$$

The boundaries of $\mathscr{B}_{0}$ and $\mathscr{B}$ are denoted by $\partial \mathscr{B}_{0}$ and $\partial \mathscr{B}$ respectively.

The deformation gradient tensor $\mathbf{A}$ is defined by

$$
\mathbf{A}=\operatorname{Grad} \boldsymbol{x},
$$

where Grad denotes the gradient operator with respect to $\mathbf{X}$, and is subject to $\operatorname{det} \mathbf{A}>0$. Polar decomposition of $\mathbf{A}$ yields

$$
\mathbf{A}=\mathbf{R} \mathbf{U}=\mathbf{V R},
$$

where $\mathbf{R}$ is a proper orthogonal tensor and $\mathbf{U}$ and $\mathbf{V}$ are positive definite symmetric tensors (respectively the right and left stretch tensors).

We may represent $\mathbf{U}$ and $\mathbf{V}$ in the spectral forms

$$
\begin{aligned}
& \mathbf{U}=\lambda_{1} \mathbf{u}^{(1)} \otimes \mathbf{u}^{(1)}+\lambda_{2} \mathbf{u}^{(2)} \otimes \mathbf{u}^{(2)}+\lambda_{3} \mathbf{u}^{(3)} \otimes \mathbf{u}^{(3)}, \\
& \mathbf{V}=\lambda_{1} \mathbf{v}^{(1)} \otimes \mathbf{v}^{(1)}+\lambda_{2} \mathbf{v}^{(2)} \otimes \mathbf{v}^{(2)}+\lambda_{3} \mathbf{v}^{(3)} \otimes \mathbf{v}^{(3)},
\end{aligned}
$$

where $\lambda_{1}, \lambda_{2}, \lambda_{3}$ are the principal stretches, $\left(\mathbf{u}^{(1)}, \mathbf{u}^{(2)}, \mathbf{u}^{(3)}\right)$ and $\left(\mathbf{v}^{(1)}, \mathbf{v}^{(2)}, \mathbf{v}^{(3)}\right)$ are two sets of orthonormal vectors defining respectively the Lagrangean and Eulerian principal directions (i.e., the principal axes of the Lagrangean and Eulerian strain ellipsoids), and

$$
\mathbf{v}^{(i)}=\mathbf{R} \mathbf{u}^{(i)}, \quad i=1,2,3 .
$$

It follows from (9)-(11) that

$$
\mathbf{A}=\lambda_{1} \mathbf{v}^{(1)} \otimes \mathbf{u}^{(1)}+\lambda_{2} \mathbf{v}^{(2)} \otimes \mathbf{u}^{(2)}+\lambda_{3} \mathbf{v}^{(3)} \otimes \mathbf{u}^{(3)} .
$$


For aǹ incompressible material

$$
\operatorname{det} \mathbf{A} \equiv \operatorname{det} \mathbf{U} \equiv \lambda_{1} \lambda_{2} \lambda_{3}=1
$$

for each point of $\mathscr{B}_{0}$.

For an isotropic elastic material the nominal stress tensor $\mathbf{S}$ may be written

$$
\mathbf{S}=\mathbf{T R}^{T}
$$

analogously to (9), where $\mathbf{T}$ is the (symmetric) Biot stress tensor and $T$ denotes the transpose of a tensor (see, for example, [3] and [4]). Since the material is isotropic (relative to $\mathscr{B}_{0}$ ), $\mathbf{T}$ is coaxial with $\mathbf{U}$ and hence we may write

$$
\mathbf{T}=t_{1} \mathbf{u}^{(1)} \otimes \mathbf{u}^{(1)}+t_{2} \mathbf{u}^{(2)} \otimes \mathbf{u}^{(2)}+t_{3} \mathbf{u}^{(3)} \otimes \mathbf{u}^{(3)},
$$

where $t_{1}, t_{2}, t_{3}$ are the principal Biot stresses, and

$$
\mathbf{S}=t_{1} \mathbf{u}^{(1)} \otimes \mathbf{v}^{(1)}+t_{2} \mathbf{u}^{(2)} \otimes \mathbf{v}^{(2)}+t_{3} \mathbf{u}^{(3)} \otimes \mathbf{v}^{(3)} .
$$

If the elastic material possesses a strain-energy function $W$ per unit reference volume then

$$
\mathbf{S}=\partial W / \partial \mathbf{A} .
$$

For $W$ to be objective (i.e., indifferent to superimposed rigid-body rotations) we must have

$$
W(\mathbf{A}) \equiv W(\mathbf{U})
$$

and then

$$
\mathbf{T}=\partial W / \partial \mathbf{U}
$$

Further, for an isotropic elastic material, $W$ depends on $\mathbf{U}$ only through $\lambda_{1}, \lambda_{2}, \lambda_{3}$, and is indifferent to interchange of any pair of $\lambda_{1}, \lambda_{2}, \lambda_{3}$. In this case we write

$$
W\left(\lambda_{1}, \lambda_{2}, \lambda_{3}\right)=W\left(\lambda_{1}, \lambda_{3}, \lambda_{2}\right)=W\left(\lambda_{3}, \lambda_{1}, \lambda_{2}\right),
$$

and then

$$
t_{i}=\partial W / \partial \lambda_{i}, \quad i=1,2,3 .
$$

For an incompressible material, equation (13) applies and equations (17), (19), and (21) are replaced by

$$
\begin{gathered}
\mathbf{S}=\partial W / \partial \mathbf{A}-p \mathbf{A}^{-1}, \\
\mathbf{T}=\partial W / \partial \mathbf{U}-p \mathbf{U}^{-1}, \\
t_{i}=\partial W / \partial \lambda_{i}-p \lambda_{i}^{-1}, \quad i=1,2,3,
\end{gathered}
$$

respectively, where $p$ is a Lagrange multiplier.

Let $\left(X_{1}, X_{2}, X_{3}\right)$ and $\left(x_{1}, x_{2}, x_{3}\right)$ denote rectangular Cartesian components of $\mathbf{X}$ and $\mathbf{x}$ respectively. Henceforth we restrict attention to plane problems in which $x_{1}, x_{2}$ depend 
only on $X_{1}, X_{2}$, and $x_{3}=\lambda_{3} X_{3}$, where $\lambda_{3}$ is a constant. We may then represent the vectors $\mathbf{u}^{(i)}$ and $\mathbf{v}^{(i)}, i=1,2,3$, in terms of their Cartesian components:

$$
\begin{aligned}
& \mathbf{u}^{(1)}=\left(\cos \theta_{L}, \sin \theta_{L}, 0\right), \quad \mathbf{u}^{(2)}=\left(-\sin \theta_{L}, \cos \theta_{L}, 0\right), \quad \mathbf{u}^{(3)}=(0,0,1), \\
& \mathbf{v}^{(1)}=\left(\cos \theta_{E}, \sin \theta_{E}, 0\right), \quad \mathbf{v}^{(2)}=\left(-\sin \theta_{E}, \cos \theta_{E}, 0\right), \quad \mathbf{v}^{(3)}=(0,0,1) .
\end{aligned}
$$

The labels ' $L$ ' and ' $E$ ' refer to 'Lagrangean' and 'Eulerian' respectively, and $\theta_{L}$ and $\theta_{E}$ describe the orientation of the Lagrangean and Eulerian principal directions in the considered plane (being measured in the anticlockwise sense from the $X_{1}$-axis).

From (12), (16), and (25) it follows that the non-vanishing Cartesian components of $\mathbf{A}$ and $\mathbf{S}$ are given by

$$
\begin{gathered}
A_{11}=\lambda_{1} \cos \theta_{L} \cos \theta_{E}+\lambda_{2} \sin \theta_{L} \sin \theta_{E}, \\
A_{12}=\lambda_{1} \sin \theta_{L} \cos \theta_{E}-\lambda_{2} \cos \theta_{L} \sin \theta_{E}, \\
A_{21}=\lambda_{1} \cos \theta_{L} \sin \theta_{E}-\lambda_{2} \sin \theta_{L} \cos \theta_{E}, \\
A_{22}=\lambda_{1} \sin \theta_{L} \sin \theta_{E}+\lambda_{2} \cos \theta_{L} \cos \theta_{E}, \\
A_{33}=\lambda_{3}, \\
S_{11}=t_{1} \cos \theta_{L} \cos \theta_{E}+t_{2} \sin \theta_{L} \sin \theta_{E}, \\
S_{12}=t_{1} \cos \theta_{L} \sin \theta_{E}-t_{2} \sin \theta_{L} \cos \theta_{E}, \\
S_{21}=t_{1} \sin \theta_{L} \cos \theta_{E}-t_{2} \cos \theta_{L} \sin \theta_{E}, \\
S_{22}=t_{1} \sin \theta_{L} \sin \theta_{E}+t_{2} \cos \theta_{L} \cos \theta_{E}, \\
\quad S_{33}=t_{3} .
\end{gathered}
$$

3. The governing equations. For the plane deformation considered above the equilibrium equation may be written in the form

$$
\frac{\partial S_{11}}{\partial X_{1}}+\frac{\partial S_{21}}{\partial X_{2}}=0, \quad \frac{\partial S_{12}}{\partial X_{1}}+\frac{\partial S_{22}}{\partial X_{2}}=0
$$

when there are no body forces. Substitution of the expressions (28) into (30) followed by elimination of terms involving $\cos \theta_{E}$ and $\sin \theta_{E}$ then yields the equations

$$
\begin{gathered}
\left(\cos \theta_{L} \frac{\partial}{\partial X_{1}}+\sin \theta_{L} \frac{\partial}{\partial X_{2}}\right) t_{1}+t_{1}\left(-\sin \theta_{L} \frac{\partial}{\partial X_{1}}+\cos \theta_{L} \frac{\partial}{\partial X_{2}}\right) \theta_{L} \\
-t_{2}\left(-\sin \theta_{L} \frac{\partial}{\partial X_{1}}+\cos \theta_{L} \frac{\partial}{\partial X_{2}}\right) \theta_{E}=0 \\
\left(-\sin \theta_{L} \frac{\partial}{\partial X_{1}}+\cos \theta_{L} \frac{\partial}{\partial X_{2}}\right) t_{2}+t_{1}\left(\cos \theta_{L} \frac{\partial}{\partial X_{1}}+\sin \theta_{L} \frac{\partial}{\partial X_{2}}\right) \theta_{E} \\
-t_{2}\left(\cos \theta_{L} \frac{\partial}{\partial X_{1}}+\sin \theta_{L} \frac{\partial}{\partial X_{2}}\right) \theta_{L}=0 .
\end{gathered}
$$

This prompts the introduction of (orthogonal) Lagrangean curvilinear coordinates $(\xi, \eta)$ such that

$$
\begin{array}{ll}
\partial X_{1} / \partial s_{\xi}=\cos \theta_{L}, & \partial X_{1} / \partial s_{\eta}=-\sin \theta_{L}, \\
\partial X_{2} / \partial s_{\xi}=\sin \theta_{L}, & \partial X_{2} / \partial s_{\eta}=\cos \theta_{L}
\end{array}
$$


and

$$
\begin{aligned}
& \partial s_{\xi} / \partial X_{1}=\cos \theta_{l,}, \quad \partial s_{\xi} / \partial X_{2}=\sin \theta_{L}, \\
& \partial s_{\eta} / \partial X_{1}=-\sin \theta_{l,}, \quad \partial s_{\eta} / \partial X_{2}=\cos \theta_{l,},
\end{aligned}
$$

analogously to (4) and (5), where $s_{\xi}$ and $s_{\eta}$ are arclength parameters associated with $\xi$ and $\eta$. The equilibrium equations (31) now take on the form ${ }^{1}$

$$
\begin{aligned}
& \frac{\partial t_{1}}{\partial s_{\xi}}+t_{1} \frac{\partial \theta_{l}}{\partial s_{\eta}}-t_{2} \frac{\partial \theta_{E}}{\partial s_{\eta}}=0, \\
& \frac{\partial t_{2}}{\partial s_{\eta}}-t_{2} \frac{\partial \theta_{l}}{\partial s_{\xi}}+t_{1} \frac{\partial \theta_{l}}{\partial s_{\xi}}=0,
\end{aligned}
$$

with $t_{1}, t_{2}, \theta_{l}$, and $\theta_{E}$ regarded as functions of the independent variables $(\xi, \eta)$.

When the constitutive law is given in the form (21) then (34) may be rewritten with $\lambda_{1}$, $\lambda_{2}, \theta_{l}$, and $\theta_{E}$ as the dependent variables. If the deformation $\mathrm{X}$ is known then the associated values of $\lambda_{1}, \lambda_{2}, \theta_{I}$, and $\theta_{E}$ are uniquely determined by the gradient $\mathbf{A}$ (subject to $0 \leqslant \theta_{l} \leqslant \pi / 2,0 \leqslant \theta_{E} \leqslant \pi / 2$ ), but, in general, an $\mathbf{A}$ with in-plane components (26) constructed from given values of $\lambda_{1}, \lambda_{2}, \theta_{l}$, and $\theta_{l}$ need not be the gradient of a deformation function $\boldsymbol{X}$. To ensure that $\mathbf{A}$ is a deformation gradient we require that the compatibility equations

$$
\frac{\partial A_{22}}{\partial X_{1}}-\frac{\partial A_{21}}{\partial X_{2}}=0, \quad \frac{\partial A_{12}}{\partial X_{1}}-\frac{\partial A_{11}}{\partial X_{2}}=0
$$

hold.

Comparison of (35) with (30) and (26) with (28) shows that (35) can be recast immediately as equations for $\lambda_{1}, \lambda_{2}, \theta_{L}$, and $\theta_{E}$, namely ${ }^{2}$

$$
\begin{aligned}
& \frac{\partial \lambda_{2}}{\partial s_{\xi}}+\lambda_{2} \frac{\partial \theta_{L}}{\partial s_{\eta}}-\lambda_{1} \frac{\partial \theta_{L}}{\partial s_{\eta}}=0, \\
& \frac{\partial \lambda_{1}}{\partial s_{\eta}}-\lambda_{1} \frac{\partial \theta_{L}}{\partial s_{\xi}}+\lambda_{2} \frac{\partial \theta_{L}}{\partial s_{\xi}}=0 .
\end{aligned}
$$

Through (21), Eqs. (34) and (36) form a set of four first-order partial differential equations for $\lambda_{1}, \lambda_{2}, \theta_{L}$, and $\theta_{E}$ when the material has no internal constraints, and, by (24), for one of $\lambda_{1}$ and $\lambda_{2}$ together with $p, \theta_{l}$, and $\theta_{E}$ when the material is incompressible. Equations (34) form a hyperbolic system when $\theta_{l}$ and $\theta_{l}$ are known, $(\xi, \eta)$ being characteristic coordinates associated with families of characteristic curves locally tangential to $\mathbf{u}^{(1)}$ and $\mathbf{u}^{(2)}$ and defined by

$$
\xi=\xi\left(X_{1}, X_{2}\right)=\text { constant }, \quad \eta=\eta\left(X_{1}, X_{2}\right)=\text { constant }
$$

\footnotetext{
'In a recent paper [8] equations equivalent to (34) have been derived.

'Equations equivalent to (36) were given in [7].
} 
in any plane section $X_{3}=$ constant of $\mathscr{B}_{0}$, subject to (32) or (33). Let such a section be denoted by $\overline{\mathscr{B}}_{0}$ and its curvilinear boundary by $\partial \overline{\mathscr{B}}_{0}$.

The tangent to a characteristic $\eta=$ constant is given by

$$
d X_{2} / d X_{1}=\tan \theta_{l}
$$

and that to $\xi=$ constant by

$$
d X_{2} / d X_{1}=-\cot \theta_{L} .
$$

Equally, (36) form a similar hyperbolic system when $\theta_{L}$ and $\theta_{E}$ are known. However, when taken together as equations for $\theta_{L}, \theta_{E}, \lambda_{1}$, and $\lambda_{2},(34)$ and (36) are not in general hyperbolic. Indeed, if the original equations for $x_{1}$ and $x_{2}$ are (strongly) elliptic, as is often assumed, then so are Eqs. (34) and (36) jointly. In this case the coordinates $(\xi, \eta)$ are not associated with characteristics, but merely with the Lagrangean principal directions.

The formulation of a boundary-value problem is complete when a pair of suitable boundary conditions is prescribed on $\partial \overline{\mathscr{B}}_{0}$. As we shall see in Sect. 4, such a pair may be recast as two equations linking $\lambda_{1}, \lambda_{2}, \theta_{L}$, and $\theta_{E}$ (or $\lambda_{1}, p, \theta_{L}$, and $\theta_{E}$, as appropriate) on $\partial \overline{\mathscr{B}}_{0}$ (or its image under (37)).

\section{Boundary conditions.}

(a) Boundary condition of traction. Let $\mathbf{N}$ denote the unit outward normal to $\partial \overline{\mathscr{B}}_{0}$. Then, by (16) with (25), we may write the boundary traction $\tau$ as

$$
\tau=\mathbf{S}^{T} \mathbf{N} \equiv t_{1}\left(\mathbf{N} \cdot \mathbf{u}^{(1)}\right) \mathbf{v}^{(1)}+t_{2}\left(\mathbf{N} \cdot \mathbf{u}^{(2)}\right) \mathbf{v}^{(2)}
$$

per unit length of $\partial \overline{\mathscr{B}}_{0}$ for the plane problem under consideration. The traction on a plane $X_{3}=$ constant is $t_{3} \mathbf{v}^{(3)}$.

Let $\mathbf{N}$ have Cartesian components $(-\sin \theta, \cos \theta, 0)$ and the tangent vector $\mathbf{M}$ to $\partial \overline{\mathscr{B}}_{0}$ have corresponding components $(\cos \theta, \sin \theta, 0)$. Then (40) yields

$$
\begin{aligned}
& t_{1} \sin \left(\theta_{L}-\theta\right) \cos \theta_{E}-t_{2} \cos \left(\theta_{L}-\theta\right) \sin \theta_{E}=\tau_{1}, \\
& t_{1} \sin \left(\theta_{L}-\theta\right) \sin \theta_{E}+t_{2} \cos \left(\theta_{L}-\theta\right) \cos \theta_{E}=\tau_{2},
\end{aligned}
$$

where $\tau_{1}, \tau_{2}$ are the Cartesian components of $\tau$, which, together with $\theta$, are known as functions of $X_{1}$ and $X_{2}$ on $\partial \overline{\mathscr{B}}_{0}$ (in the case of dead load tractions).

We also have $t_{3}=\partial W / \partial \lambda_{3}$, and for plane strain this equation specifies the normal stress required to maintain fixed $\lambda_{3}$.

(b) Boundary condition of place. If $x_{\alpha}=\chi_{\alpha}\left(X_{1}, X_{2}\right), \alpha=1,2$, is prescribed on $\partial \overline{\mathscr{B}}_{0}$ then

$$
(\mathbf{M} \cdot \mathrm{Grad}) \mathbf{\chi} \equiv \mathbf{A M} \equiv \mathbf{R U M}
$$

is known and directed along the tangent to the deformed boundary (i.e., $\mathbf{M}$ is an embedded vector). We may write the boundary condition as

$$
\lambda_{1}\left(\mathbf{M} \cdot \mathbf{u}^{(1)}\right) \mathbf{v}^{(1)}+\lambda_{2}\left(\mathbf{M} \cdot \mathbf{u}^{(2)}\right) \mathbf{v}^{(2)}=\mathbf{w},
$$

with $\mathbf{w}$ prescribed on $\partial \overline{\mathscr{B}}_{0}$. In Cartesian components this takes the form

$$
\begin{aligned}
& \lambda_{1} \cos \left(\theta_{L_{L}}-\theta\right) \cos \theta_{E}-\lambda_{2} \sin \left(\theta_{L_{-}}-\theta\right) \sin \theta_{E}=w_{1}, \\
& \lambda_{1} \cos \left(\theta_{L}-\theta\right) \sin \theta_{E}+\lambda_{2} \sin \left(\theta_{L}-\theta\right) \cos \theta_{E}=w_{2},
\end{aligned}
$$

analogously to (41). 
In principle the four dependent variables can be found from the above equations and boundary conditions. The two boundary conditions interconnect these variables at each point of the boundary $\partial \overline{\mathscr{B}}_{0}$. The analytical solution of the equations is illustrated in Sect. 6 for a simple problem, while details of the numerical solution of boundary-value problems are reserved for a subsequent paper.

Once $\lambda_{1}, \lambda_{2}, \theta_{L}$, and $\theta_{E}$ have been determined, the deformation function is obtained by integration of $d \mathbf{x}=\mathbf{A} d \mathbf{X}$ using (26) and (32).

5. Eulerian formulation. Here we provide an alternative formulation of the governing equations based on the current configuration with coordinate curves along the Eulerian principal axes. Analogously to (32) we have

$$
\begin{array}{ll}
\frac{\partial x_{1}}{\partial s_{\xi^{*}}}=\cos \theta_{E}, & \frac{\partial x_{1}}{\partial s_{\eta^{*}}}=-\sin \theta_{E}, \\
\frac{\partial x_{2}}{\partial s_{\xi^{*}}}=\sin \theta_{E}, & \frac{\partial x_{2}}{\partial s_{\eta^{*}}}=\cos \theta_{E},
\end{array}
$$

where the current curvilinear coordinates $\left(\xi^{*}, \eta^{*}\right)$ are such that

$$
\frac{\partial s_{\xi^{*}}}{\partial s_{\xi}}=\lambda_{1}, \quad \frac{\partial s_{\eta^{*}}}{\partial s_{\eta}}=\lambda_{2}, \quad \frac{\partial s_{\xi^{*}}}{\partial s_{\eta}}=\frac{\partial s_{\eta^{*}}}{\partial s_{\xi}}=0 .
$$

In terms of the principal components $\sigma_{1}, \sigma_{2}$ of the Cauchy stress tensor $J^{-1} \mathbf{A S}$, the equilibrium equations (34) may be rewritten as

$$
\begin{aligned}
& \frac{\partial \sigma_{1}}{\partial s_{\xi^{*}}}+\left(\sigma_{1}-\sigma_{2}\right) \frac{\partial \theta_{E}}{\partial s_{\eta^{*}}}=0, \\
& \frac{\partial \sigma_{2}}{\partial s_{\eta^{*}}}+\left(\sigma_{1}-\sigma_{2}\right) \frac{\partial \theta_{E}}{\partial s_{\xi^{*}}}=0,
\end{aligned}
$$

which, in different notation, are the same as (1). The compatibility equations (36) may similarly be expressed in terms of $\xi^{*}$ and $\eta^{*}$.

In the linear theory $\left(\xi^{*}, \eta^{*}\right)$ are identified with $(\xi, \eta)$ and we introduce the principal infinitesimal strains $e_{1}=\lambda_{1}-1, e_{2}=\lambda_{2}-1$ with $\lambda_{3}$ fixed as unity. From (36), we then obtain

$$
\begin{aligned}
& \frac{\partial}{\partial s_{\xi}}\left(\theta_{L}-\theta_{E}\right)=\frac{\partial e_{1}}{\partial s_{\eta}}-\left(e_{1}-e_{2}\right) \frac{\partial \theta_{E}}{\partial s_{\xi}}, \\
& \frac{\partial}{\partial s_{\eta}}\left(\theta_{L}-\theta_{E}\right)=-\frac{\partial e_{2}}{\partial s_{\xi}}+\left(e_{1}-e_{2}\right) \frac{\partial \theta_{E}}{\partial s_{\eta}},
\end{aligned}
$$

correct to the first order in $e_{1}, e_{2}$, and their derivatives. This means that, to this order, $\theta_{L}$ cannot be identified with $\theta_{E}$.

Equations (46), with $\left(\xi^{*}, \eta^{*}\right)$ replaced by $(\xi, \eta)$, and (47), together with the constitutive relations

$$
\sigma_{\alpha}=2 \mu e_{\alpha}+\lambda\left(e_{1}+e_{2}\right), \quad \alpha=1,2,
$$

for a linear isotropic elastic material, where $\lambda$ and $\mu$ are the Lamé moduli, form a coupled system of equations for $e_{1}, e_{2}, \theta_{L}$, and $\theta_{E}$. Note that $e_{1}+e_{2}$ also satisfies Laplace's 
equation, which can be written

$$
\left(\frac{\partial^{2}}{\partial s_{\xi}^{2}}+\frac{\partial^{2}}{\partial s_{\eta}^{2}}\right)\left(e_{1}+e_{2}\right)+\frac{\partial}{\partial s_{\xi}}\left(e_{1}+e_{2}\right) \frac{\partial \theta_{E}}{\partial s_{\eta}}-\frac{\partial}{\partial s_{\eta}}\left(e_{1}+e_{2}\right) \frac{\partial \theta_{E}}{\partial s_{\xi}}=0
$$

6. Illustration: flexure of a rectangular block. We consider a plane strain problem with $\lambda_{3}=1$ for a body whose undeformed plane section is defined by

$$
-A \leqslant X_{1} \leqslant A, \quad-B \leqslant X_{2} \leqslant B \text {. }
$$

Suppose this section is deformed into a sector of a circular annulus in such a way that straight lines $X_{1}=$ constant become circles $r=$ constant and straight lines $X_{2}=$ constant become radial lines $\theta=$ constant, where $r$ and $\theta$ are plane polar coordinates. For an incompressible material the deformation is described by

$$
r^{2}=\beta+2 X_{1} / \alpha, \quad \theta=\alpha X_{2},
$$

where $\alpha$ and $\beta$ are constants (to be determined by the boundary conditions). For detailed discussion of this deformation we refer to [4]-[6].

It is easily shown from the above that $\theta_{L}=0, \theta_{E}=\theta$, and $\lambda_{2}=\lambda_{1}^{-1}=\alpha r$. From (32) we deduce that the coordinates $(\xi, \eta)$ can be identified with $\left(X_{1}, X_{2}\right)$. The compatibility equations (36) are automatically satisfied and the equilibrium equations reduce to

$$
\partial t_{1} / \partial X_{1}=\alpha t_{2}, \quad \partial t_{2} / \partial X_{2}=0
$$

On $X_{1}=$ constant the traction is $t_{1}$ in the radial direction, and on $X_{2}=$ constant the traction is $t_{2}$ in the $\theta$-direction.

We introduce the notation $\lambda=\lambda_{1}=1 / \alpha r$ and write

$$
\hat{W}(\lambda)=W\left(\lambda, \lambda^{-1}, 1\right)
$$

so that, by (24),

$$
\lambda_{1} t_{1}-\lambda_{2} t_{2}=\lambda \hat{W}^{\prime}(\lambda)
$$

where the primes denotes differentiation with respect to $\lambda$.

On changing the independent variable $X_{1}$ to $\lambda$ and eliminating $t_{2}$ between $(50)_{1}$ and (51), we obtain

$$
\lambda d t_{1} / d \lambda+t_{1}=\hat{W}^{\prime}(\lambda)
$$

and hence

$$
\lambda t_{1}=\hat{W}(\lambda)+\gamma
$$

where $\gamma$ is a constant. The stress $t_{2}$ is then expressed as a function of $\lambda$ by means of (51) and (52).

At this stage there are three unknown constants, $\alpha, \beta, \gamma$, to be determined.

Suppose that we impose the boundary conditions

$$
t_{1}=0 \quad \text { on } X_{1}= \pm A \text {. }
$$

Then, from (52) we obtain

$$
-\gamma=\hat{W}\left(\lambda_{+}\right)=\hat{W}\left(\lambda_{-}\right)
$$

where

$$
\lambda_{ \pm}=\left(\alpha^{2} \beta \pm 2 \alpha A\right)^{1 / 2}
$$

thus providing two equations linking $\alpha, \beta$, and $\gamma$. 
Because of (53) it follows from (50) that the total load on the boundaries $X_{2}= \pm B$ vanishes. The moment $M$ of the tractions on $X_{2}= \pm B$ about the origin $r=0$ is given by

$$
M=\int_{-A}^{A} r t_{2} d X_{1} .
$$

Expressed in terms of the independent variable $\lambda$, this can be rewritten as

$$
M=\frac{1}{\alpha^{2}} \int_{\lambda}^{\lambda_{+}} \lambda^{-3}\{\hat{W}(\lambda)+\gamma\} d \lambda
$$

or, equivalently, as

$$
M=\frac{1}{2 \alpha^{2}} \int_{\lambda}^{\lambda_{+}} \lambda^{-2} \hat{W}^{\prime}(\lambda) d \lambda .
$$

This provides a third equation relating $\alpha, \beta$, and $\gamma$ to the boundary tractions.

For the neo-Hookean or Mooney strain-energy functions we have

$$
\hat{W}=\frac{1}{2} \mu\left(\lambda^{2}+\lambda^{-2}-2\right)
$$

and the following explicit results are obtained. Equations (54) yield

$$
\begin{gathered}
\beta^{2}=\left(1+4 \alpha^{2} A^{2}\right) / \alpha^{4}, \\
\gamma=\mu\left[1-2 \alpha A-\sqrt{1+4 \alpha^{2} A^{2}}\right],
\end{gathered}
$$

while the relationship between $M$ and $\alpha$ is calculated from (56) as

$$
M=\frac{\mu}{2 \alpha^{2}} \ln \left[2 \alpha A+\sqrt{1+4 \alpha^{2} A^{2}}\right]-\frac{\mu A}{\alpha} \sqrt{1+4 \alpha^{2} A^{2}} .
$$

Acknowledgment. The writer is grateful to Dr. G. Moore, Brunel University, for discussions concerning the numerical solution of the equations derived here.

\section{REFERĘNCES}

[1] H. J. Jessop, Photoelasticity, in Handbuch der Physik, Vol. VI (Edited by S. Flügge), Springer, 1958

[2] A. Franek, J. Kratochvil and L. Travnicek, ZAMM 63, 156-158 (1983)

[3] R. W. Ogden, Inequalities associated with the inversion of elastic stress-deformation relations and their implications, Math. Proc. Cambridge Philos. Soc. 81, 313-324 (1977)

[4] R. W. Ogden, Non-linear elastic deformations, Ellis Horwood, 1984

[5] A. E. Green and W. Zerna, Theoretical elasticitt, Oxford University Press, 1968

[6] A. E. Green and J. E. Adkins, Large elastic deformations, Oxford University Press, 1970

[7] R. T. Shield, The rotation associated with large strains, SIAM J. Appl. Math. 25, 483-491 (1973)

[8] A. Franek, J. Kratochvil, and L. Travnicek, Inhomogeneous inverse problem in finite elasticity, J. Elasticity 14, $363-372(1984)$ 